\title{
Design Parameter and Anisotropy Constant Ratio for Heat-Assisted Magnetic Recording
}

\author{
T. Kobayashi, Y. Isowaki, and Y. Fujiwara \\ Graduate School of Engineering, Mie Univ., 1577 Kurimamachiya-cho, Tsu 514-8507, Japan
}

A design guideline for heat-assisted magnetic recording (HAMR) is presented with a view to obtaining a minimized anisotropy constant ratio $K_{\mathrm{u}} / K_{\mathrm{bulk}}$ for media by using a model calculation. We investigated the relationship between the design parameters and the limiting factors of HAMR design. The parameters, namely the writing temperature $T_{\mathrm{w}}$ and recording layer thickness $h$, are related to the medium thermal stability factor of the limiting factor. In addition, inter-grain exchange coupling $J$ is related to the statistical thermal stability factor. HAMR combined with shingled magnetic recording (SHAMR) has the advantage of reducing the medium thermal gradient, and increasing $T_{\mathrm{w}}$ is also effective in increasing the heat-transfer thermal gradient of the limiting factor. The combination of increasing $h$ and SHAMR or increasing $J$ and SHAMR can successfully reduce $K_{\mathrm{u}} / K_{\mathrm{bulk}}$. Furthermore, $K_{\mathrm{u}} / K_{\text {bulk }}$ can be reduced by the combination of increasing $T_{\mathrm{w}}$ and SHAMR.

Key words: heat-assisted magnetic recording, media design, anisotropy constant ratio, thermal stability factor, thermal gradient

\section{Introduction}

Heat-assisted magnetic recording (HAMR) has been proposed with the aim of solving the trilemma problem $^{1)}$ of magnetic recording. HAMR is a recording method in which the medium is heated to reduce the coercivity at the time of writing.

We have already reported the design method for $\left.\mathrm{HAMR}^{2}, 3\right)$. We have introduced a new parameter, the anisotropy constant ratio $K_{\mathrm{u}} / K_{\text {bulk }}$, which is the intrinsic ratio of film anisotropy constant $K_{\mathrm{u}}$ to bulk $K_{\mathrm{u}}{ }^{2}$. If the $K_{\mathrm{u}} / K_{\text {bulk }}$ values for two media are identical, the difficulties involved in preparing those media are the same, even if the $K_{\mathrm{u}}$ values are different. On the other hand, increasing $K_{\mathrm{u}} / K_{\text {bulk }}$ is a challenging task. Therefore, it is necessary to design a medium with a smaller $K_{\mathrm{u}} / K_{\text {bulk }}$. The many relationships between design parameters and $K_{\mathrm{u}} / K_{\text {bulk }}$ have been revealed ${ }^{3)}$. We have reported the dependence of $K_{\mathrm{u}} / K_{\text {bulk }}$ on one parameter change. As a result, we find that increasing the writing temperature $T_{\mathrm{w}}$ is only effective for reducing $K_{\mathrm{u}} / K_{\text {bulk }}$ when we compare the $K_{\mathrm{u}} / K_{\text {bulk }}$ value calculated using standard parameter values. Although the combination of more than two parameters may be expected to reduce $K_{\mathrm{u}} / K_{\text {bulk }}$, it is difficult to examine all parameter combinations. Our method is a model calculation, and it is easy to understand the physical meanings of the parameters.

In this study, we investigate the relationship between the design parameters and limiting factors of HAMR design with the aim of reducing $K_{\mathrm{u}} / K_{\text {bulk }}$, and we provide the examination results of $K_{\mathrm{u}} / K_{\text {bulk }}$ for the combination of more than two parameters.

\section{Media Design Method}

From the following four conditions, we estimated whether or not the media have the potential for HAMR. That is, information stability (1) during 10 years of archiving, (2) on the trailing side located 1 bit from the writing position during writing, (3) in adjacent tracks during rewriting, and (4) under the main pole during rewriting 2,3 ).

(1) The first condition, namely the information stability during 10 years of archiving, is expressed as

$$
\frac{K_{\mathrm{u}}\left(T_{\mathrm{a}}\right) V_{\mathrm{m}}}{k T_{\mathrm{a}}} \geq \mathrm{TSF}_{10},
$$

where $K_{\mathrm{u}}\left(T_{\mathrm{a}}\right) V_{\mathrm{m}} / k T_{\mathrm{a}}$ is a medium thermal stability factor, $T_{\mathrm{a}}$ is the ambient temperature (assumed to be $330 \mathrm{~K}$ ), which is the maximum working temperature of the hard drive, $V_{\mathrm{m}}$ is the grain volume for the mean grain size, $k$ is the Boltzmann constant, and $\mathrm{TSF}_{10}$ is the statistical thermal stability factor during 10 years of archiving.

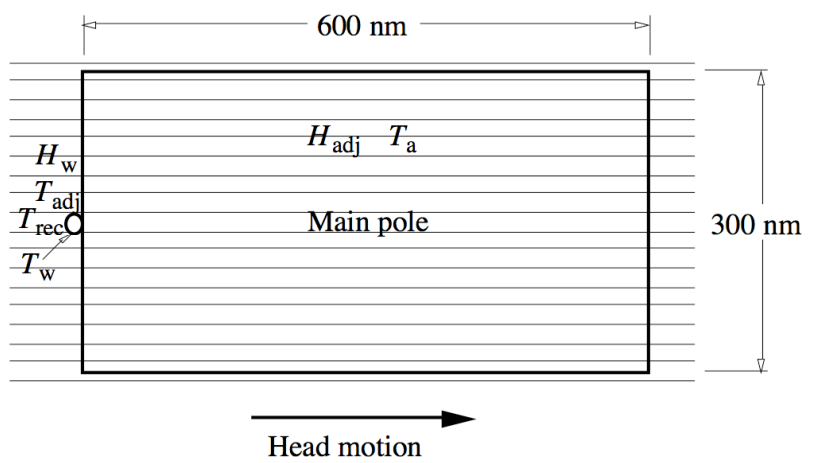

Fig. 1 Schematic illustration of writing-head configuration.

Figure 1 shows the writing-head configuration. It is assumed that the main-pole size of the head is $600 \mathrm{~nm}$ (down-track direction) $\times 300 \mathrm{~nm} \quad$ (cross-track 
direction), and the writing position is located on the trailing side of the main pole. The maximum temperature under the main pole is $T_{\mathrm{a}}$.

(2) The second condition, namely the information stability on the trailing side during writing, is expressed as

$$
\frac{\Delta T}{\Delta x}=\frac{T_{\mathrm{w}}-T_{\mathrm{rec}}}{\Delta x} \leq \frac{\partial T}{\partial x},
$$

where $\Delta T / \Delta x$ is the medium thermal gradient for the down-track direction, $T_{\mathrm{w}}$ is the writing temperature, and $T_{\text {rec }}$ is the maximum temperature at which the information on the trailing side can be held during writing as shown in Fig. 1. $\Delta x$ is the distance from the position of $T_{\mathrm{w}}$ to that of $T_{\text {rec }}$, and $\partial T / \partial x$ is the heat-transfer thermal gradient for the down-track direction.

(3) The third condition, namely the information stability in adjacent tracks during rewriting, is expressed as

$$
\frac{\Delta T}{\Delta y}=\frac{T_{\mathrm{w}}-T_{\text {adj }}}{\Delta y} \leq \frac{\partial T}{\partial y},
$$

where $\Delta T / \Delta y$ is the medium thermal gradient for the cross-track direction, and $T_{\text {adj }}$ is the maximum temperature at which the information in adjacent tracks can be held during rewriting as shown in Fig. 1. $\Delta y$ is the distance from the position of $T_{\mathrm{w}}$ to that of $T_{\text {adj }}$, and $\partial T / \partial y$ is the heat-transfer thermal gradient for the cross-track direction. $\Delta T / \Delta x$ and $\Delta T / \Delta y$ are the minimum thermal gradients required by the medium for information stability. $\partial T / \partial x$ and $\partial T / \partial y$ are the thermal gradients calculated by a heat-transfer simulation ${ }^{3)}$.

(4) The fourth condition, namely the information stability under the main pole during rewriting, is expressed as

$$
H_{\text {adj }} \geq H_{\mathrm{w}} \text {, }
$$

where $H_{\text {adj }}$ is the maximum head field that can hold the information under the main pole, and $H_{\mathrm{w}}$ is the writing field at the position of $T_{\mathrm{w}}$ as shown in Fig. 1.

The details have been reported elsewhere ${ }^{3)}$.

The HAMR media were designed to obtain the minimum $K_{\mathrm{u}} / K_{\text {bulk }}$ value using the procedure shown in Fig. $2^{3)}$. First, the design parameters and $K_{\mathrm{u}} / K_{\text {bulk }}$ = 1 were set. Four HAMR conditions (1), (2), (3), and (4) were examined after determining the composition of the recording layer. If there are some margins for all four conditions, $K_{\mathrm{u}} / K_{\text {bulk }}$ can be reduced. When one of the four conditions reaches the limit, the minimum $K_{\mathrm{u}} / K_{\text {bulk }}$ value can be determined. That condition becomes a limiting factor.

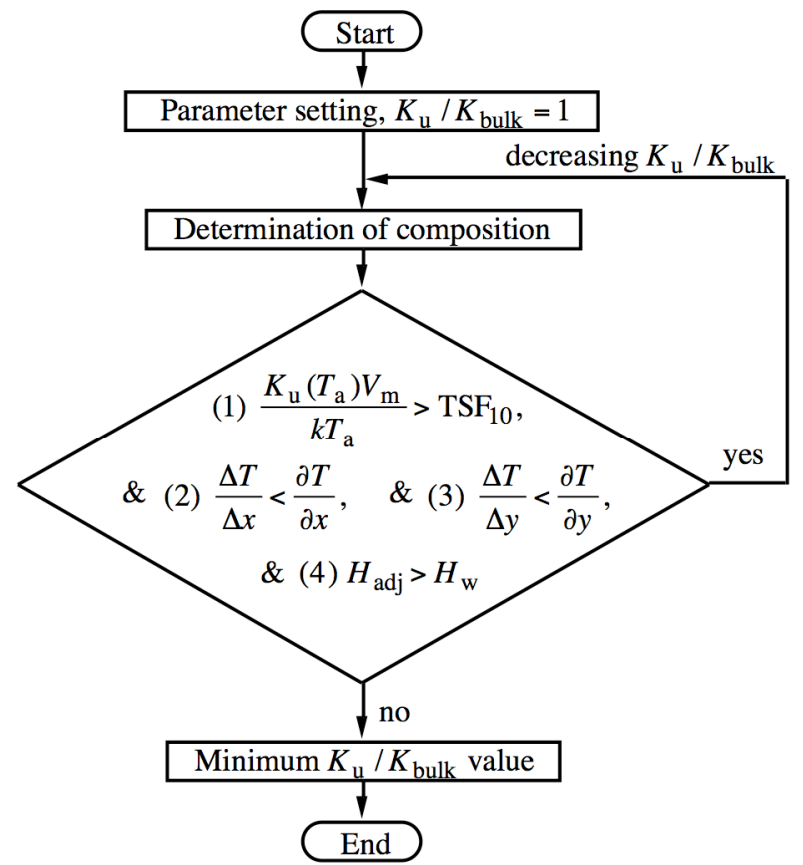

Fig. 2 HAMR media design procedure for obtaining the minimum $K_{\mathrm{u}} / K_{\text {bulk }}$ value $^{3)}$.

The medium was assumed to be granular. The standard parameter values of the media design are summarized in Table 1. The bit area $S$ is fixed, and $S$ is the product $d_{\mathrm{B}} \times d_{\mathrm{T}}$ of the bit pitch $d_{\mathrm{B}}$ and the track pitch $d_{\mathrm{T}}$. The method for determining $d_{\mathrm{T}} / d_{\mathrm{B}}$ was reported in a previous paper ${ }^{2}$. The arrangement of the grains is not considered. The effective track width $d_{\mathrm{ET}}$ has been explained in a previous paper ${ }^{3)}$. The standard medium consists of four layers, that is, a recording layer $\mathrm{RL}$ (Fe-Pt base, thickness $h=8 \mathrm{~nm}$ ), interlayer $1 \mathrm{IL} 1$ ( $\mathrm{MgO}$ base, $5 \mathrm{~nm})$, interlayer $2 \mathrm{IL} 2(\mathrm{Cr}$ base, $10 \mathrm{~nm})$, and a heat-sink layer HSL $(\mathrm{Cu}$ base, 30 $\mathrm{nm}) . D_{\mathrm{m}}$ is the mean grain size, and $V_{\mathrm{m}}=D_{\mathrm{m}}{ }^{2} \times h$. The inter-grain exchange coupling $J^{4)}$ is a newly introduced design parameter. A comparison of magnetic recording (MR) method was carried out of HAMR and HAMR combined with shingled magnetic recording (SHAMR). The light-spot diameter $d_{\mathrm{L}}$ and the heat-spot diameter $d_{\mathrm{w}}$ have also been defined in previous papers ${ }^{2,3)}$.

Figure 3 (a) is a schematic illustration of the area near the writing position for HAMR. The writing field $H_{\mathrm{w}}$ is applied to a wide area including the writing position. The circle denoted by $T_{\mathrm{w}}$ is an isotherm of $T_{\mathrm{w}}$, and $d_{\mathrm{w}}$ is the heat-spot diameter. The white regions indicate upward or downward magnetization, and the gray regions indicate the magnetization transition. The transition region spreads to adjacent tracks as a result of rewriting operations on the $i$ th track.

On the other hand, the maximum rewriting number for SHAMR is considerably smaller than that for HAMR since the tracks are laid to overlap each other as shown in Fig. 3 (b). 
Table 1 Standard parameter values of media design.

\begin{tabular}{l|c}
\hline User areal density (Tbpsi) & 4 \\
Bit area $S\left(\mathrm{~nm}^{2}\right)$ & 140 \\
Effective track width $d_{\mathrm{ET}}(\mathrm{nm})$ & 10 \\
Ambient temperature $T_{\mathrm{a}}(\mathrm{K})$ & 330 \\
\hline Writing temperature $T_{\mathrm{w}}(\mathrm{K})$ & 500 \\
Grain number per bit $n$ (grain / bit) & 4 \\
RL thickness $h(\mathrm{~nm})$ & 8 \\
Standard deviation of grain size $\sigma_{\mathrm{D}} / D_{\mathrm{m}}(\%)$ & 10 \\
Inter - grain exchange coupling $J\left(\mathrm{erg} / \mathrm{cm}^{2}\right)$ & 0 \\
MR method & $\mathrm{HAMR}$ \\
Light - spot diameter $d_{\mathrm{L}}(\mathrm{nm})$ & 9.0 \\
Heat - spot diameter $d_{\mathrm{w}}(\mathrm{nm})$ & 10 \\
Linear velocity $v(\mathrm{~m} / \mathrm{s})$ & 10 \\
Thermal conductivity of $\mathrm{IL} 1 K(\mathrm{~W} /(\mathrm{cmK}))$ & 0.5 \\
\hline
\end{tabular}

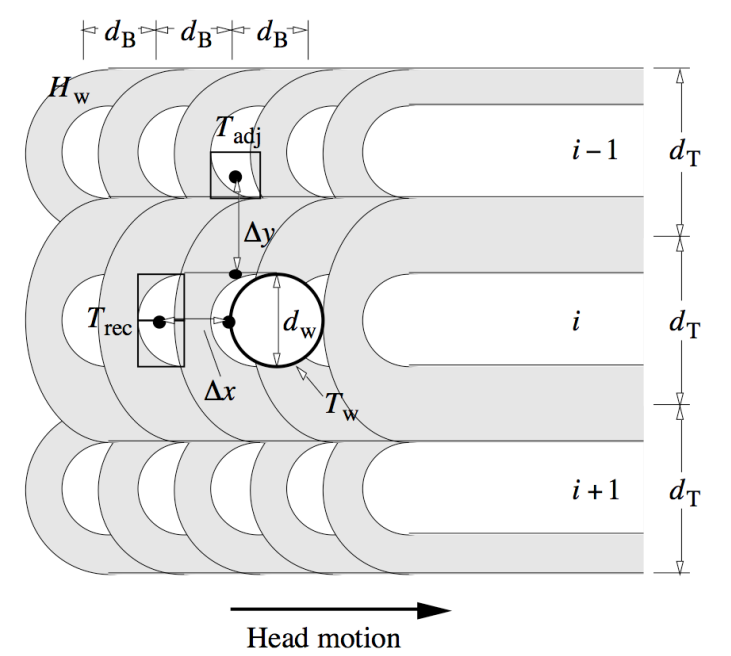

(a)

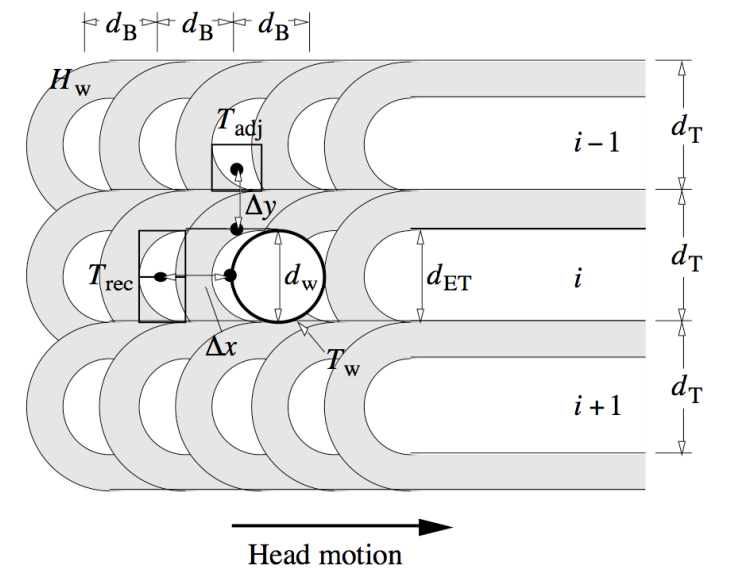

(b)

Fig. 3 Schematic illustrations of writing position for (a) HAMR and (b) SHAMR.

\section{Design Parameters and Limiting Factors}

We have reported the dependence of $K_{\mathrm{u}} / K_{\text {bulk }}$ on one parameter change ${ }^{3)}$. As a result, increasing $T_{\mathrm{w}}$ is only effective for reducing $K_{\mathrm{u}} / K_{\text {bulk }}$ when we compare the $K_{\mathrm{u}} / K_{\text {bulk }}$ value calculated using standard parameter values. Increasing $J$ from 0 to $0.19 \mathrm{erg} / \mathrm{cm}^{2}$ has little effect. Although the combination of more than two parameters may be expected to reduce $K_{\mathrm{u}} / K_{\text {bulk }}$, it is difficult to examine all parameter combinations. Therefore, we investigated the relationship between the parameters and the limiting factors. Conditions (2) and (3) can be combined as

$$
\frac{\Delta T}{\Delta x}=\frac{\Delta T}{\Delta y} \leq \frac{\partial T}{\partial x}=\frac{\partial T}{\partial y}
$$

since $\partial T / \partial x \approx \partial T / \partial y^{2}$. Condition (4) has some margins for all the cases we examined. Therefore, the major limiting factors in the media design are condition (I) given by Eq. (1) $\left(K_{\mathrm{u}}\left(T_{\mathrm{a}}\right) V_{\mathrm{m}} / k T_{\mathrm{a}} \geq \mathrm{TSF}_{10}\right)$ and condition (II) given by Eq. (5) (hereafter, shown as $\Delta T / \Delta x=\Delta T / \Delta y \equiv \Delta T / \Delta x(y) \quad, \quad \partial T / \partial x=\partial T / \partial y$ $\equiv \partial T / \partial x(y)$, and $\Delta T / \Delta x(y) \leq \partial T / \partial x(y))$.

\subsection{Condition (I) - increasing $K_{\mathrm{u}}\left(T_{\mathrm{a}}\right) V_{\mathrm{m}} / k T_{\mathrm{a}}$}

$K_{\mathrm{u}}\left(T_{\mathrm{a}}\right) V_{\mathrm{m}} / k T_{\mathrm{a}}$ must be increased or $\mathrm{TSF}_{10}$ decreased to relax condition (I) given by Eq. (1) $K_{\mathrm{u}}\left(T_{\mathrm{a}}\right) V_{\mathrm{m}} / k T_{\mathrm{a}} \geq \mathrm{TSF}_{10}$. First, we discuss the parameter for increasing $K_{\mathrm{u}}\left(T_{\mathrm{a}}\right)$.

Figure 4 (a) shows the dependence of $K_{\mathrm{u}}(300 \mathrm{~K})$ on Curie temperature $T_{\mathrm{c}}$, in which the calculation parameter is $K_{\mathrm{u}} / K_{\text {bulk }}$. The dotted line shows the $K_{\mathrm{u}}(300 \mathrm{~K})$ value that satisfies $K_{\mathrm{u}}\left(T_{\mathrm{a}}\right) V_{\mathrm{m}} / k T_{\mathrm{a}}=\mathrm{TSF}_{10}$. Although the $K_{\mathrm{u}}\left(T_{\mathrm{a}}\right)$ value that satisfies $K_{\mathrm{u}}\left(T_{\mathrm{a}}\right) V_{\mathrm{m}} / k T_{\mathrm{a}}=\mathrm{TSF}_{10}$ is independent of $T_{\mathrm{c}}$, the $K_{\mathrm{u}}(300 \mathrm{~K})$ value that satisfies $K_{\mathrm{u}}\left(T_{\mathrm{a}}\right) V_{\mathrm{m}} / k T_{\mathrm{a}}=\mathrm{TSF}_{10}$ increases as $T_{\mathrm{c}}$ decreases, and it diverges at $T_{\mathrm{a}}=330$ $K$. The intersections of the solid lines and the dotted line show the $K_{\mathrm{u}} / K_{\text {bulk }}$ dependence that satisfies $K_{\mathrm{u}}\left(T_{\mathrm{a}}\right) V_{\mathrm{m}} / k T_{\mathrm{a}}=\mathrm{TSF}_{10}$ on $T_{\mathrm{c}}$. Therefore, the required $K_{\mathrm{u}} / K_{\text {bulk }}$ can be reduced by increasing $T_{\mathrm{c}}$, and then, $K_{\mathrm{u}} / K_{\text {bulk }}$ can be reduced by increasing $T_{\mathrm{w}}$ as shown in Fig. 4 (b). In other words, if $K_{\mathrm{u}} / K_{\text {bulk }}$ is constant, increasing $T_{\mathrm{w}}$ is effective for increasing $K_{\mathrm{u}}\left(T_{\mathrm{a}}\right)$. If $T_{\mathrm{w}}$ is low, the extra $K_{\mathrm{u}} / K_{\text {bulk }}$ is needed to realize HAMR.

Furthermore, $K_{\mathrm{u}} / K_{\text {bulk }}$ is a function of the heat-transfer thermal gradient $\partial T / \partial x(y)$ as shown in Fig. 4 (c). As $\partial T / \partial x(y)$ increases, $K_{\mathrm{u}} / K_{\text {bulk }}$ first becomes lower, and then becomes constant with respect to $\partial T / \partial x(y)$. In the range where $K_{\mathrm{u}} / K_{\text {bulk }}$ changes, the limiting factor is condition (II) $\Delta T / \Delta x(y)$ $\leq \partial T / \partial x(y)$. And it is condition (I) $K_{\mathrm{u}}\left(T_{\mathrm{a}}\right) V_{\mathrm{m}} / k T_{\mathrm{a}}$ $\geq \mathrm{TSF}_{10}$ in the range where $K_{\mathrm{u}} / K_{\text {bulk }}$ is constant. The constant $K_{\mathrm{u}} / K_{\text {bulk }}$ value in Fig. 4 (c) corresponds to that in Fig. $4(\mathrm{~b})$. Although increasing $T_{\mathrm{w}}$ is effective for reducing $K_{\mathrm{u}} / K_{\text {bulk }}$ (Fig. $4(\mathrm{~b})$ ), a higher $\partial T / \partial x(y)$ is needed to obtain a lower $K_{\mathrm{u}} / K_{\text {bulk }}$ (Fig. 4 (c)). The closed circles show the $K_{\mathrm{u}} / K_{\text {bulk }}$ values and their $\partial T / \partial x(y)$ values calculated for the standard medium structure and the standard thermal constants ${ }^{2)}$. Increasing $T_{\mathrm{w}}$ is effective for reducing $K_{\mathrm{u}} / K_{\text {bulk }}$ in consideration of calculated $\partial T / \partial x(y)$. 


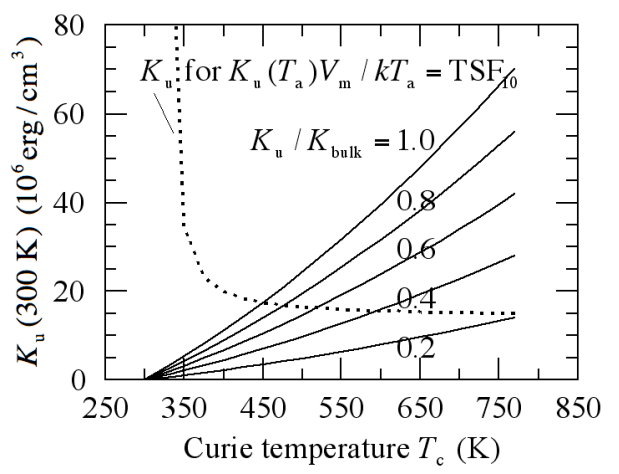

(a)

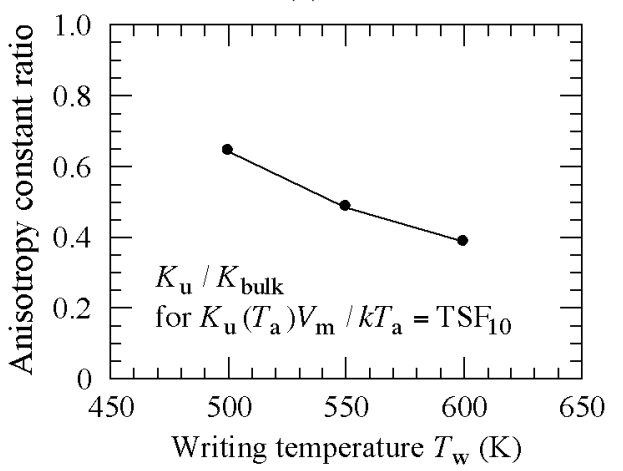

(b)

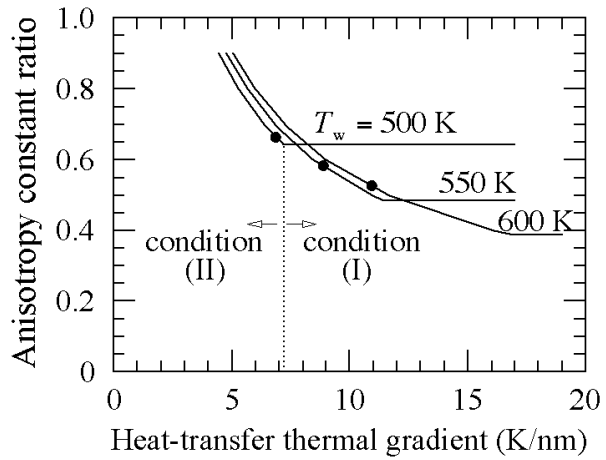

(c)

Fig. 4 (a) Dependence of anisotropy constant $K_{\mathrm{u}}(300 \mathrm{~K})$ on Curie temperature $T_{\mathrm{c}}$, (b) dependence of anisotropy constant ratio $K_{\mathrm{u}} / K_{\text {bulk }}$ on writing temperature $T_{\mathrm{w}}$, and (c) dependence of $K_{\mathrm{u}} / K_{\text {bulk }}$ on heat-transfer thermal gradient $\partial T / \partial x(y)$.

Next, we discuss the parameter for increasing $V_{\mathrm{m}}$.

One way of increasing $V_{\mathrm{m}}=D_{\mathrm{m}}{ }^{2} \times h$ is to reduce the grain number per bit $n$. Although $\mathrm{TSF}_{10}$ becomes larger as $n$ becomes smaller ${ }^{4)}, \quad D_{\mathrm{m}}=\sqrt{S / n}-\Delta$ increases as $n$ decreases where $S$ is the bit area and $\Delta=1 \mathrm{~nm}$ is the non-magnetic spacing between grains (e.g., $D_{\mathrm{m}}=4.92 \mathrm{~nm}$ for $n=4$ ). The solid line, in the range where $K_{\mathrm{u}} / K_{\text {bulk }}$ is constant in Fig. 5, becomes noticeably low as $n$ decreases. In that range, the limiting factor is condition (I) as mentioned above. Therefore, reducing $n$ relaxes condition (I) through increasing $V_{\mathrm{m}}$. Furthermore, the closed circles can also be reduced as $n$ decreases. Therefore, reducing $n$ is effective for reducing $K_{\mathrm{u}} / K_{\text {bulk }}$. When we compare (a) $T_{\mathrm{w}}=500 \mathrm{~K}$ and (b) $550 \mathrm{~K}$, the $n$ dependence of the $K_{\mathrm{u}} / K_{\text {bulk }}$ values of the closed circles is reduced by increasing $T_{\mathrm{w}}$ since the limiting factor becomes condition (II).

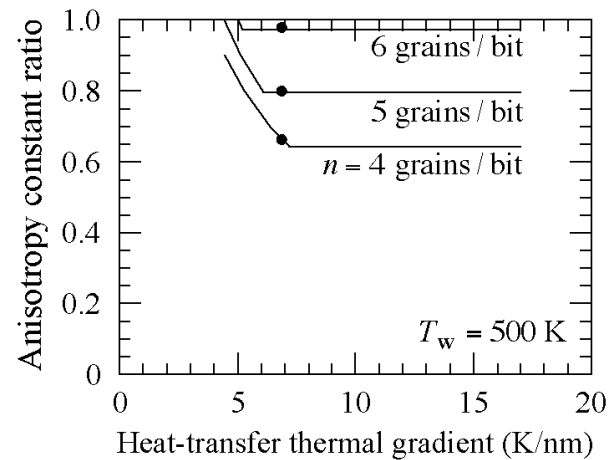

(a)

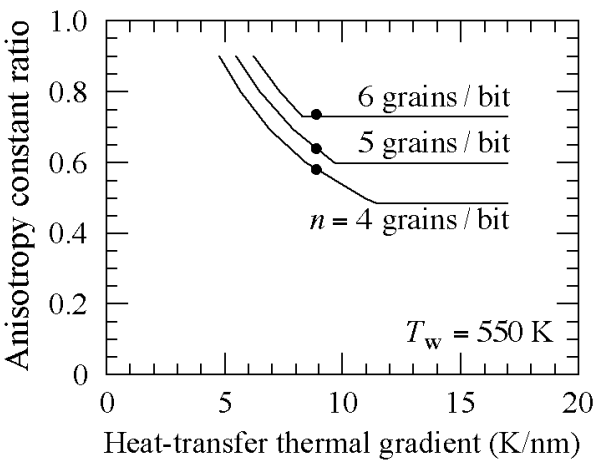

(b)

Fig. 5 Dependence of $K_{\mathrm{u}} / K_{\text {bulk }}$ on $\partial T / \partial x(y)$ at (a) $T_{\mathrm{w}}=500 \mathrm{~K}$ and (b) $550 \mathrm{~K}$ (calculation parameter: grain number per bit $n$ ).

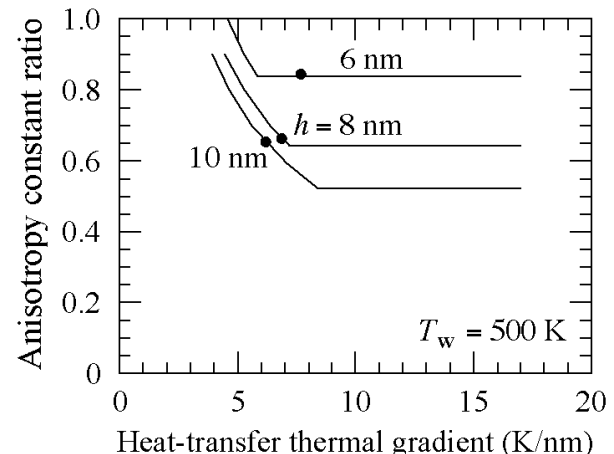

(a)

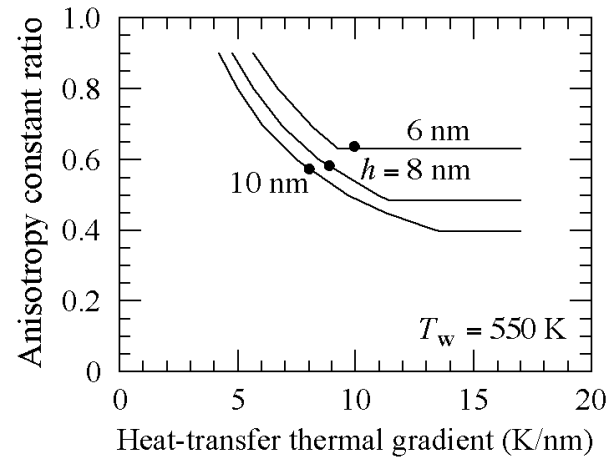

(b)

Fig. 6 Dependence of $K_{\mathrm{u}} / K_{\text {bulk }}$ on $\partial T / \partial x(y)$ at (a) $T_{\mathrm{w}}=500 \mathrm{~K}$ and (b) $550 \mathrm{~K}$ (calculation parameter: $\mathrm{RL}$ thickness $h$ ). 
Another way of increasing $V_{\mathrm{m}}=D_{\mathrm{m}}{ }^{2} \times h$ is to increase the RL thickness $h$. It can also be seen from the solid lines where $K_{\mathrm{u}} / K_{\text {bulk }}$ is constant in Fig. 6 that increasing $h$ relaxes condition (I). However, when $h$ increases from the standard value of 8 to $10 \mathrm{~nm}$, the $K_{\text {u }} / K_{\text {bulk }}$ values of the closed circles are almost the same for both (a) $T_{\mathrm{w}}=500 \mathrm{~K}$ and (b) $550 \mathrm{~K}$ since the limiting factor is condition (II), and $\partial T / \partial x(y)$ is decreased by increasing $h$ due to the adiabatic effect of $\mathrm{RL}^{2)}$. If $\Delta T / \Delta x(y)$ can be decreased or $\partial T / \partial x(y)$ can be increased in some way, increasing $h$ will be effective for reducing $K_{\mathrm{u}} / K_{\text {bulk }}$. The way of decreasing $\Delta T / \Delta x(y)$ will be discussed in 3.3 , and the combination of the parameters will be discussed in $\mathbf{3 . 5}$.

\subsection{Condition (I) - decreasing $\mathrm{TSF}_{10}$}

$\mathrm{TSF}_{10}$ must be decreased to relax condition (I) given by Eq. (1) $K_{\mathrm{u}}\left(T_{\mathrm{a}}\right) V_{\mathrm{m}} / k T_{\mathrm{a}} \geq \mathrm{TSF}_{10}$.

One way of decreasing $\mathrm{TSF}_{10}$ is to reduce the standard deviation of the grain size $\sigma_{\mathrm{D}} / D_{\mathrm{m}}{ }^{4}$. Decreasing $\sigma_{\mathrm{D}} / D_{\mathrm{m}}$ relaxes condition (I) as seen from the solid lines in Fig. 7. Furthermore, the $K_{\mathrm{u}} / K_{\text {bulk }}$ values of the closed circles can be reduced by decreasing $\sigma_{\mathrm{D}} / D_{\mathrm{m}}$ as shown in Fig. 7 (a). However, it seems that $\sigma_{\mathrm{D}} / D_{\mathrm{m}}$ cannot be reduced from the standard value of $10 \%$.

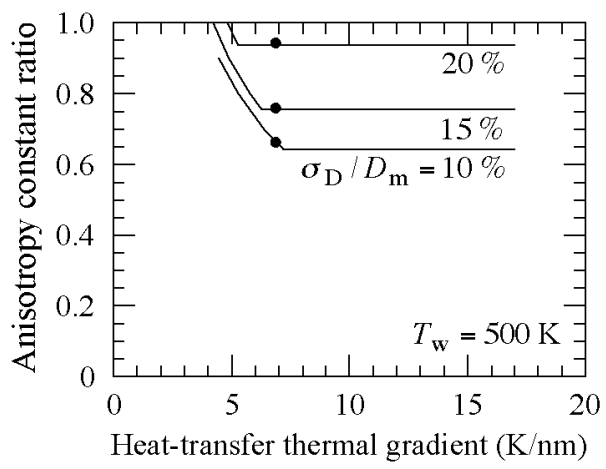

(a)

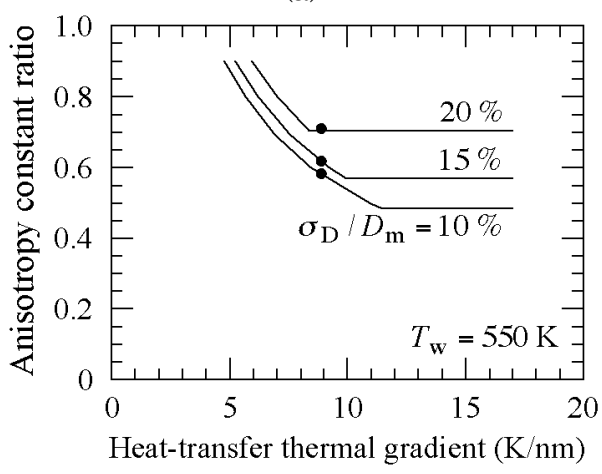

(b)

Fig. 7 Dependence of $K_{\mathrm{u}} / K_{\text {bulk }}$ on $\partial T / \partial x(y)$ at (a) $T_{\mathrm{w}}=500 \mathrm{~K}$ and (b) $550 \mathrm{~K}$ (calculation parameter: standard deviation of grain size $\left.\sigma_{\mathrm{D}} / D_{\mathrm{m}}\right)$.

In Fig. 7 (b), it should be noted that the difference between the $K_{\mathrm{u}} / K_{\text {bulk }}$ values of the closed circles $\left(\sigma_{\mathrm{D}} / D_{\mathrm{m}}=10 \%\right.$ and $\left.15 \%\right)$ is very small. If $T_{\mathrm{w}}$ can be increased, a relatively large $\sigma_{\mathrm{D}} / D_{\mathrm{m}}$ value may be allowable.

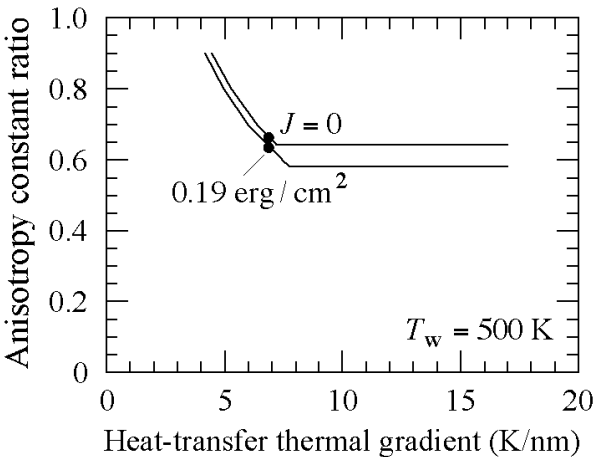

(a)

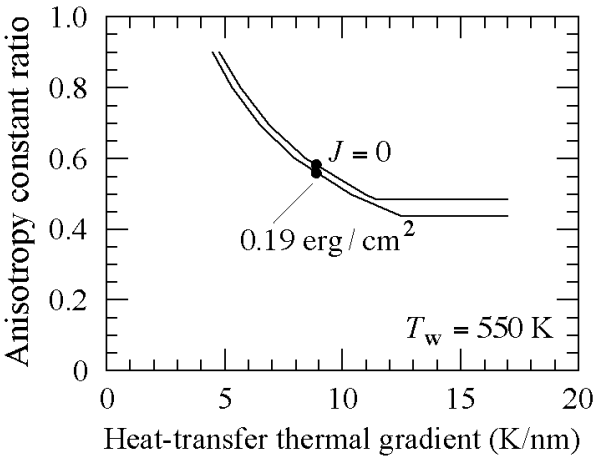

(b)

Fig. 8 Dependence of $K_{\mathrm{u}} / K_{\text {bulk }}$ on $\partial T / \partial x(y)$ at (a) $T_{\mathrm{w}}=500 \mathrm{~K}$ and (b) $550 \mathrm{~K}$ (calculation parameter: inter-grain exchange coupling $J$ ).

Although another way of decreasing $\mathrm{TSF}_{10}$ is to introduce inter-grain exchange coupling $J^{4)}$ as seen from the solid lines in Fig. 8. $J=0.19 \mathrm{erg} / \mathrm{cm}^{2}$ corresponds to an inter-grain exchange coupling $e_{\mathrm{m}}$ of 1.14) for $D_{\mathrm{m}}$. The inter-grain exchange coupling $e$ makes the statistical thermal stability factor $e$ times larger. It has little effect on reducing the $K_{\mathrm{u}} / K_{\text {bulk }}$ values of the closed circles since the limiting factor is condition (II). As seen from Fig. 8 (b), increasing $T_{\mathrm{w}}$ has little effect for the same reason. If $\Delta T / \Delta x(y)$ can be decreased or $\partial T / \partial x(y)$ can be increased in some way, introducing $J$ will also be effective for reducing $K_{\mathrm{u}} / K_{\text {bulk }}$. The combination of the parameters will be discussed in $\mathbf{3 . 5}$.

\subsection{Condition (II) - decreasing $\Delta T / \Delta x(y)$}

$\Delta T / \Delta x(y)$ must be decreased or $\partial T / \partial x(y)$ increased to relax condition (II) given by Eq. (5) $\Delta T / \Delta x(y) \leq \partial T / \partial x(y)$.

Introducing SHAMR is effective for decreasing $\Delta T / \Delta x(y)$ as seen from the solid lines where $K_{\mathrm{u}} / K_{\text {bulk }}$ changes in Fig. 9 (a). $T_{\text {adj }}$ in Eq. (3) for SHAMR is higher than that for HAMR since the maximum rewriting number for SHAMR is considerably smaller than that for $\mathrm{HAMR}^{3}$. However, the $K_{\mathrm{u}} / K_{\text {bulk }}$ values of the closed circles are almost the same since the limiting factor is condition (I). If we 
can find a way to increase $K_{\mathrm{u}}\left(T_{\mathrm{a}}\right) V_{\mathrm{m}} / k T_{\mathrm{a}}$ or decrease $\mathrm{TSF}_{10}$, introducing SHAMR will be effective for reducing $K_{\mathrm{u}} / K_{\text {bulk }}$. Figure 9 (b) shows the results for $T_{\mathrm{w}}=550 \mathrm{~K}$. Since the limiting factor can be changed to condition (II), introducing SHAMR becomes effective.

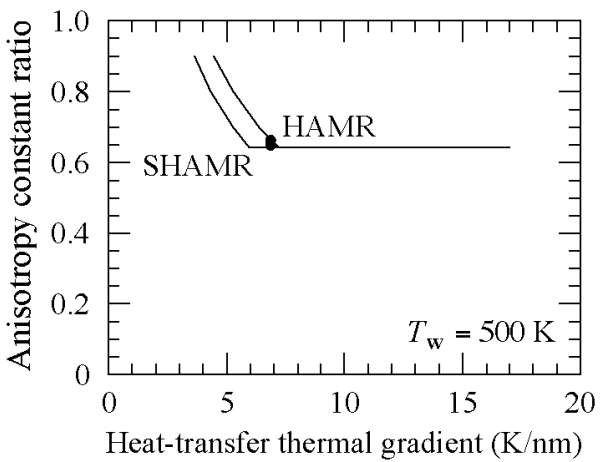

(a)

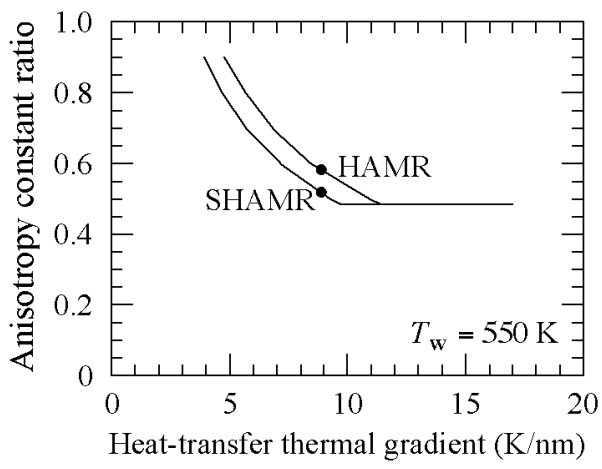

(b)

Fig. 9 Dependence of $K_{\mathrm{u}} / K_{\text {bulk }}$ on $\partial T / \partial x(y)$ at (a) $T_{\mathrm{w}}=500 \mathrm{~K}$ and (b) $550 \mathrm{~K}$ (calculation parameter: HAMR and SHAMR).

\subsection{Condition (II) - increasing $\partial T / \partial x(y)$}

Although increasing $T_{\mathrm{w}}$ is effective for increasing $\partial T / \partial x(y)$ as mentioned above in 3.1, $\partial T / \partial x(y)$ can be changed very little by changing the parameters, namely the light-spot diameter $d_{\mathrm{L}}$, the heat-spot diameter $d_{\mathrm{w}}$, and the linear velocity $v$ 3). If the thermal conductivity $K$ of IL1 decreases from 0.5 $\mathrm{W} /(\mathrm{cmK}), \partial T / \partial x(y)$ becomes smaller as shown in a previous paper ${ }^{3)}$. One possibility for increasing $\partial T / \partial x(y)$ involves examining a media structure with a large $\partial T / \partial x(y)$.

\subsection{Combination of parameters}

The combination of one parameter that relaxes condition (I) and another that relaxes condition (II) may be useful for reducing $K_{\mathrm{u}} / K_{\text {bulk }}$.

When we replaced parameter $h=8 \mathrm{~nm}$ with 10 $\mathrm{nm}, K_{\mathrm{u}} / K_{\text {bulk }}$ changed from 0.66 to 0.65 , and when only SHAMR was utilized, $K_{\mathrm{u}} / K_{\text {bulk }}$ became 0.64 at $T_{\mathrm{w}}=500 \mathrm{~K}$. Alone they had scarcely any effect on reducing $K_{\mathrm{u}} / K_{\text {bulk }}$. Next, we combined $h=10 \mathrm{~nm}$, which increases $K_{\mathrm{u}}\left(T_{\mathrm{a}}\right) V_{\mathrm{m}} / k T_{\mathrm{a}}$ of condition (I), and SHAMR, which decreases $\Delta T / \Delta x(y)$ of condition (II). Figure 10 (a) shows the result of the combination, and the resultant $K_{\mathrm{u}} / K_{\text {bulk }}$ is 0.58 . The case where $T_{\mathrm{w}}=$ $550 \mathrm{~K}$ is shown in Fig. 10 (b). When we compare HAMR and SHAMR, we find that SHAMR is more effective. This can be explained by Fig. 9 (b). On the other hand, when we compare a SHAMR of $h=8 \mathrm{~nm}$ and a SHAMR of $h=10 \mathrm{~nm}$, the $K_{\mathrm{u}} / K_{\text {bulk }}$ values are almost the same since the limiting factor is condition (II) as seen in Fig. 6.

Therefore, the design parameters of HAMR, SHAMR, $h$, and $T_{\mathrm{w}}$ are related in a complex manner in terms of reducing $K_{\mathrm{u}} / K_{\text {bulk }}$.

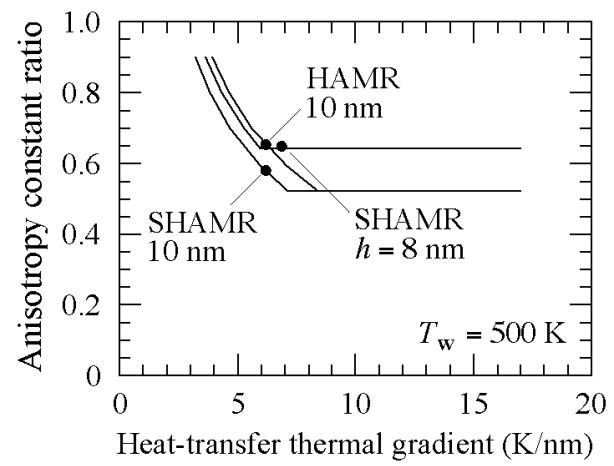

(a)

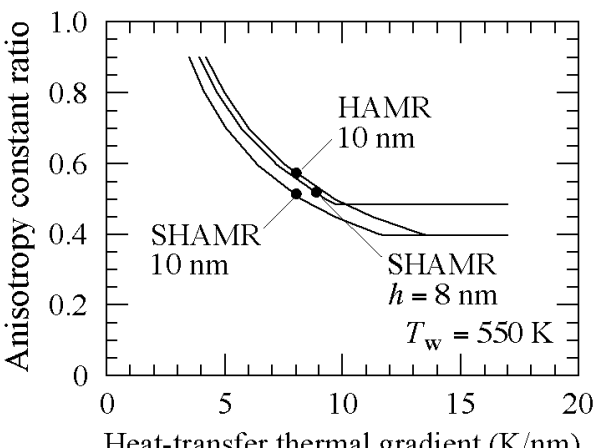

(b)

Fig. 10 Dependence of $K_{\mathrm{u}} / K_{\text {bulk }}$ on $\partial T / \partial x(y)$ at (a) $T_{\mathrm{w}}=500 \mathrm{~K}$ and (b) $550 \mathrm{~K}$ (calculation parameter: HAMR, SHAMR, and $h$ ).

Next, we consider the relationship between HAMR, SHAMR, $J$, and $T_{\mathrm{w}}$

We combined $J=0.19 \mathrm{erg} / \mathrm{cm}^{2}$, which decreases $\mathrm{TSF}_{10}$ of condition (I), and SHAMR, which decreases $\Delta T / \Delta x(y)$ of condition (II). Figure 11 (a) shows the result of the combination, and the resultant $K_{\mathrm{u}} / K_{\text {bulk }}$ is 0.58. The case of $T_{\mathrm{w}}=550 \mathrm{~K}$ is shown in Fig. 11 (b). When we compare a SHAMR of $J=0$ and a SHAMR of $J=0.19 \mathrm{erg} / \mathrm{cm}^{2}$, the $K_{\mathrm{u}} / K_{\text {bulk }}$ values are almost the same since the limiting factor is condition (II).

The results are summarized in Fig. 12. The standard $K_{\mathrm{u}} / K_{\text {bulk }}$ value (calculated using the standard parameter values) is 0.66 . The parameters $T_{\mathrm{w}}, h$, and $J$ relax condition (I) $K_{\mathrm{u}}\left(T_{\mathrm{a}}\right) V_{\mathrm{m}} / k T_{\mathrm{a}}$ $\geq \mathrm{TSF}_{10}$, and the $K_{\mathrm{u}} / K_{\text {bulk }}$ values become $0.58,0.65$, and 0.63 , respectively, by changing the parameters. And the parameters HAMR or SHAMR and $T_{\mathrm{w}}$ relax condition (II) $\Delta T / \Delta x(y) \leq \partial T / \partial x(y)$, and the $K_{\mathrm{u}} / K_{\text {bulk }}$ 
values become to be 0.64 and 0.58 , respectively.

If $h=10 \mathrm{~nm}$ and SHAMR or $J=0.19 \mathrm{erg} / \mathrm{cm}^{2}$ and SHAMR are combined, $K_{\mathrm{u}} / K_{\text {bulk }}=0.58$ can be achieved without increasing $T_{\mathrm{w}}$. A lower $T_{\mathrm{w}}$ is better from the viewpoint of the heat resistance of the writing head and/or the surface lubricant.

If $T_{\mathrm{w}}$ can be increased to $550 \mathrm{~K}$, we can expect a $K_{\mathrm{u}} / K_{\text {bulk }}$ value of 0.51 by combination with SHAMR.

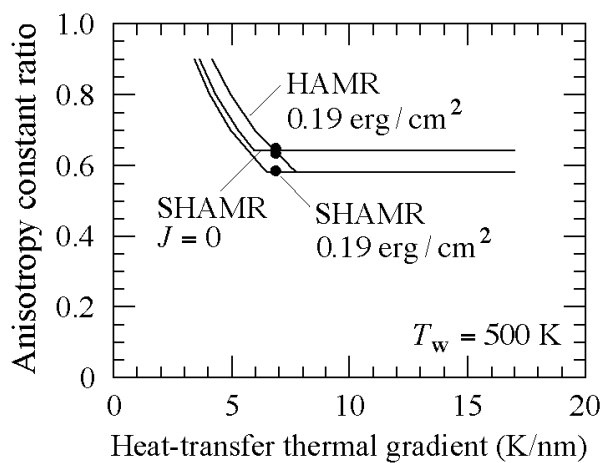

(a)

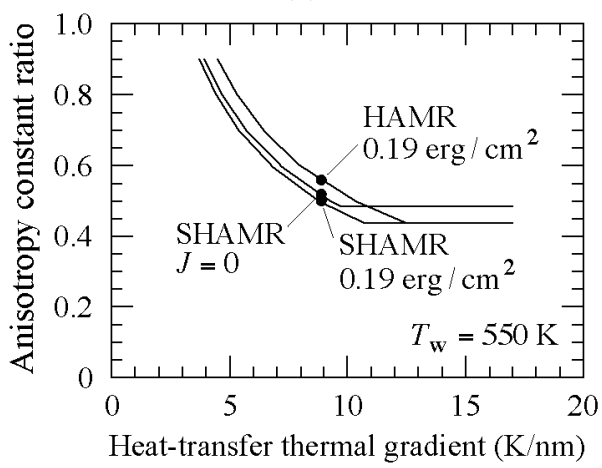

(b)

Fig. 11 Dependence of $K_{\mathrm{u}} / K_{\text {bulk }}$ on $\partial T / \partial x(y)$ at (a) $T_{\mathrm{w}}=500 \mathrm{~K}$ and (b) $550 \mathrm{~K}$ (calculation parameter: HAMR, SHAMR, and $J$ ).

$$
\begin{aligned}
& \text { Standard } K_{\mathrm{u}} / K_{\text {bulk }}=0.66 \\
& \text { (I) } \frac{K_{\mathrm{u}}\left(T_{\mathrm{a}}\right) V_{\mathrm{m}}}{k T_{\mathrm{a}}} \geq \mathrm{TSF}_{10} \text { relaxing } \\
& T_{\mathrm{w}}=550 \mathrm{~K} \leftarrow 500 \mathrm{~K} \quad 0.58 \\
& h=10 \mathrm{~nm} \leftarrow 8 \mathrm{~nm} \quad 0.65 \\
& J=0.19 \mathrm{erg} / \mathrm{cm}^{2} \leftarrow 0 \\
& \text { (II) } \frac{\Delta T}{\Delta x(y)} \leq \frac{\partial T}{\partial x(y)} \text { relaxing } \\
& \text { SHAMR } \leftarrow \text { HAMR } \\
& T_{\mathrm{w}}=550 \mathrm{~K} \leftarrow 500 \mathrm{~K}
\end{aligned}
$$

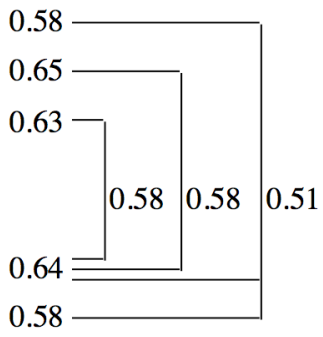

Fig. 12 Media design guideline.

\section{Conclusions}

The major limiting factors as regards obtaining the minimum anisotropy constant ratio $K_{\mathrm{u}} / K_{\text {bulk }}$ for media are (I) the information stability during 10 years of archiving and (II) the information stability on the trailing side during writing and in adjacent tracks during rewriting.

The parameters, namely the writing temperature $T_{\mathrm{w}}$, the recording layer thickness $h$, and the inter-grain exchange coupling $J$, relax condition (I). On the other hand, HAMR combined with shingled magnetic recording (SHAMR) and $T_{\mathrm{w}}$ relax condition (II).

The combination of one parameter that relaxes condition (I) and another that relaxes condition (II) is useful for reducing $K_{\mathrm{u}} / K_{\text {bulk }}$. If increasing $h$ and SHAMR or increasing $J$ and SHAMR are combined, $K_{\mathrm{u}} / K_{\text {bulk }}$ can be reduced successfully without increasing $T_{\mathrm{w}}$. If $T_{\mathrm{w}}$ can be increased, $K_{\mathrm{u}} / K_{\text {bulk }}$ will be reduced by the combination with SHAMR.

It is important for the media design to distinguish whether the limiting factor is condition (I) or (II).

Acknowledgements We acknowledge the support of the Storage Research Consortium (SRC), Japan.

\section{References}

1) S. H. Charap, P.-L. Lu, and Y. He: IEEE Trans. Magn., 33 978 (1997).

2) T. Kobayashi, Y. Isowaki, and Y. Fujiwara: J. Magn. Soc. Jpn., 39, 8 (2015).

3) T. Kobayashi, Y. Isowaki, and Y. Fujiwara: J. Magn. Soc Jpn., 39, 139 (2015).

4) Y. Isowaki, T. Kobayashi, and Y. Fujiwara: J. Magn. Soc. Jpn., 38, 1 (2014).

Received Apr. 12, 2015; Revised Jun. 17, 2015; Accepted Oct. 8, 2015 